\title{
Vortex Lattice Melting in Layered Superconductors with Periodic Columnar Pins
}

\author{
Chandan Dasgupta ${ }^{1, *}$ and Oriol T. Valls ${ }^{2, \dagger}$ \\ ${ }^{1}$ Department of Physics, Indian Institute of Science, Bangalore 560012, India \\ ${ }^{2}$ Department of Physics and Minnesota Supercomputer Institute, University of Minnesota, Minneapolis, Minnesota 55455-0149
}

\begin{abstract}
The melting of the vortex lattice in highly anisotropic, layered superconductors with commensurate, periodic columnar pins is studied in a geometry where magnetic field and columnar pins are normal to the layers. Thermodynamic properties and equilibrium density distributions are obtained from numerical minimizations of an appropriate free-energy functional. We find a line of first-order transitions that ends at a critical point as the pin concentration is increased. We quantitatively determine the location of this critical point and show that it is experimentally accessible.
\end{abstract}

Thermodynamic and transport properties of type-II superconductors in the mixed phase can be altered in a controlled manner by the introduction of artificial pinning centers. Columnar pinning in high-temperature superconductors (HTSCs), produced by damage tracks from heavy-ion bombardment, has been extensively studied experimentally $[1,2]$ and theoretically $[3,4]$. A random array of such pins leads to a glassy phase, known as Bose glass [3]. The interplay between the lattice constant of the pin array and the intervortex separation should produce [5] commensurability effects. Such effects have been observed in imaging experiments [6] and magnetization measurements [7] on thin-film superconductors with periodic arrays of artificially produced pinning centers.

In this Letter, we investigate the effects of a commensurate, periodic array of columnar pins on the vortex lattice melting transition [8] in highly anisotropic, layered, HTSCs in a magnetic field. Both the field and the columnar pins are assumed to be normal to the layers. We consider values of the field for which the pin areal density is smaller than that of the vortex lines. Although vortex lattice melting in HTSCs in the absence of pinning has been studied extensively [8], not much is known about the effects of columnar pins on this transition. Since columnar defects produce strong pinning, each defect should pin a vortex at low temperatures. However, interstitial vortices, present when there are more vortices than pins, should [4] undergo a melting transition at a temperature slightly higher than [9] the melting point of the pure vortex lattice. Evidence for such melting of interstitial vortices has been found in experiments [6,7] on thin-film superconductors with periodic pinning, but the thermodynamics of this transition has not been studied.

Since the defect-pinned vortices produce a periodic potential for the interstitial ones, the melting transition of the latter provides a physical realization of three-dimensional melting in the presence of an external periodic potential. Using density functional theory $[10,11]$ and a newly developed numerical method [12] that is uniquely suited for calculations of pinning-induced inhomogeneities in the local density, we have studied the effects of this periodic po- tential on the structure and thermodynamics of the liquid and crystalline states of a system of "pancake" vortices [8] in a highly anisotropic layered superconductor. For small concentrations of pinning centers, we find a first-order melting transition from a crystalline solid to a modulated liquid. As the pin concentration is increased, the transition temperature increases, and the latent heat and the jump in the crystalline order parameter at the transition decrease. This line of first-order transitions terminates at a quantitatively determined, experimentally accessible critical point beyond which the thermodynamic transition is replaced by a sharp crossover. We believe that this is the first reliable theoretical prediction of a continuous melting transition in an experimentally accessible three-dimensional system. Similar melting transitions are of interest in other systems such as atoms adsorbed on crystalline substrates [13], and colloidal particles in interfering laser fields [14] and arrays of optical traps [15]. Our method and results would be of relevance to these systems also.

We consider a layered superconductor with vanishingly small Josephson coupling between layers (vortices on different layers are coupled via their electromagnetic interaction only). In this limit of effectively infinite anisotropy, which is appropriate [11] for extremely anisotropic $\mathrm{Bi}$ and Tl-based compounds, the energy of a system of pancake vortices residing on the superconducting layers may be written as a sum of anisotropic two-body interactions $v(n, r)$, where $n$ is the layer separation and $r$ is the separation in the plane of the layers. The Fourier transform of $v$ is [11]

$$
\beta v(\mathbf{k})=\frac{2 \pi \Gamma \lambda^{2}\left[k_{\perp}^{2}+\left(4 / d^{2}\right) \sin ^{2}\left(k_{z} d / 2\right)\right]}{k_{\perp}^{2}\left[1+\lambda^{2} k_{\perp}^{2}+4\left(\lambda^{2} / d^{2}\right) \sin ^{2}\left(k_{z} d / 2\right)\right]},
$$

with $\Gamma \equiv \beta d \Phi_{0}^{2} / 8 \pi^{2} \lambda^{2}$ and $\beta=1 / k_{B} T$. Here, $k_{z}\left(k_{\perp}\right)$ is the component of $\mathbf{k}$ perpendicular (parallel) to the layer plane, $d$ is the layer spacing, $\lambda(T)$ is the penetration depth in the layer plane, and $\Phi_{0}$ is the flux quantum. The intralayer potential $v(n=0, r)$ is repulsive and $\propto \ln (r)$, whereas the interlayer potential $v(n \neq 0, r)$, also $\propto \ln (r)$, is attractive, weaker than the intralayer potential by the 
factor $d / \lambda$, and decreases exponentially with $n$ as $e^{-n d / \lambda}$. We use parameters appropriate to $\mathrm{Bi}_{2} \mathrm{Sr}_{2} \mathrm{CaCu}_{2} \mathrm{O}_{8}-\delta$ (BSCCO), i.e., $\lambda(T=0)=1500 \AA$ and $d=15 \AA$, and assume a two-fluid $T$ dependence of $\lambda$ with $T_{c}(0)=85 \mathrm{~K}$.

In density functional theory $[10,11,16]$, the free energy of a state in a density configuration specified by $\rho(i, \mathbf{r})$, the time averaged areal density of vortices at point $\mathbf{r}$ on the $i$ th layer, is given in terms of equilibrium correlation functions of the layered liquid of pancake vortices. We use the Ramakrishnan-Yussouff free-energy functional
[10] which is known [11] to provide a quantitatively correct description of the melting transition in our system in the absence of pinning. The presence of a commensurate array of columnar pins leads to an appropriate "external potential" term [5] in the free-energy functional. Since the potential produced by a set of straight columnar pins perpendicular to the layers is the same on every layer, the time-averaged density of vortices at any point $\mathbf{r}$ must be the same on all layers: $\rho(i, \mathbf{r})=\rho(\mathbf{r})$ for all $i$. Then, the free energy per layer may be written in a two-dimensional form:

$$
\begin{aligned}
\beta\left(F[\rho]-F_{0}\right)= & \int d^{2} r\left[\rho(\mathbf{r}) \ln \frac{\rho(\mathbf{r})}{\rho_{0}}-\delta \rho(\mathbf{r})\right]-\frac{1}{2} \int d^{2} r \int d^{2} r^{\prime} \tilde{C}\left(\left|\mathbf{r}-\mathbf{r}^{\prime}\right|\right) \delta \rho(\mathbf{r}) \delta \rho\left(\mathbf{r}^{\prime}\right) \\
& +\beta \int d^{2} r V_{p}(\mathbf{r}) \delta \rho(\mathbf{r}) .
\end{aligned}
$$

Here, $\delta \rho(\mathbf{r}) \equiv \rho(\mathbf{r})-\rho_{0}, F_{0}$ is the free energy of the uniform liquid of areal density $\rho_{0}\left(=B / \Phi_{0}\right.$ where $B$ is the magnetic induction), $V_{p}(\mathbf{r})$ is the pinning potential, and $\tilde{C}(r) \equiv \sum_{n} C(n, r)$, where $C(n, r)$ is the direct pair correlation function [16] of a layered liquid of pancake vortices. We use the results for $C(n, r)$ obtained [11] from a hypernetted chain calculation [16].

The pinning potential at point $\mathbf{r}$ is given by $\beta V_{p}(\mathbf{r})=$ $\sum_{j} V_{0}\left(\left|\mathbf{r}-\mathbf{R}_{\mathbf{j}}\right|\right)$, where the sum is over all pinning centers located at the points $\left\{\mathbf{R}_{j}\right\}$ on a plane, and $V_{0}(r)$, the potential at $\mathbf{r}$ due to a pinning center at the origin, is assumed to have the truncated parabolic form,

$$
V_{0}(r)=-\alpha \Gamma\left(1-r^{2} / r_{0}^{2}\right)
$$

for $r \leq r_{0}$ and $V_{0}(r)=0$ if $r>r_{0}$. Here, $r_{0}$ is the range and $\alpha$ is a "strength" parameter chosen to ensure that on the average a pinning center traps $\leqslant 1$ vortex in the temperature range of interest.

To find the minima of the free energy of Eq. (2), we discretize space by defining density variables $\left\{\rho_{k}\right\}$ at the sites of a periodic grid, and use a gradient descent method [12] to locate the minima of the free energy of Eq. (2) written as a function of $\left\{\rho_{k}\right\}$. The use of fast Fourier transforms in the calculation of lattice sums [17] speeds the computations and allows studies of larger systems. To accommodate a triangular lattice, we use a triangular grid with periodic boundary conditions.

We have performed our studies for $B=2$ and $3 \mathrm{kG}$. We first checked the results for crystallization without pinning. For this purpose, the computational system was one triangular lattice unit cell with lattice constant $a$ (all lengths are in units of $a_{0}$, with $\left.\pi a_{0}^{2} \equiv 1 / \rho_{0}=\Phi_{0} / B\right)$, and the spacing $h$ of the computational grid was chosen to have the values $a / n$ with $n=16,32,64$, and 128 . The free energies of the crystal obtained [18] for all these values of $n$ are essentially the same, indicating that the effects of discretization are minimal for $h \leq a / 16$. The equilibrium value $a_{m}$ of the lattice parameter $a$ was determined by finding the value of $a$ that minimizes the free energy at a given $B$ and $T$. The value of $a_{m}$ is found to be slightly higher than the spacing of a perfect triangular lattice of density $\rho_{0}$. This reflects the well-known result [8] that the density of a vortex lattice increases slightly at melting.

The free energy of the crystal crosses zero at the transition temperature $T_{c}=18.45 \mathrm{~K}$ at $B=2 \mathrm{kG}$. As expected, $T_{c}$ is slightly higher than that obtained from approximate treatments [11] of the same free energy. The entropy change $\Delta s$ per vortex is $0.29 k_{B}$, and the jump in the order parameter $m$ [ $m$ is the magnitude of the Fourier component of $\rho(\mathbf{r})$ at the shortest reciprocal lattice vector of the triangular lattice] is $\Delta m=0.52$. The Lindemann parameter calculated from the density distribution [18] at the crystalline minimum is $\mathcal{L}=0.26$ at melting. Very similar results were obtained for $B=3 \mathrm{kG}\left(T_{c}=15.10 \mathrm{~K}\right.$, $\left.\Delta s=0.28 k_{B}, \Delta m=0.52, \mathcal{L}=0.25\right)$. The close agreement of these results with those of other studies [8,11] establishes the validity and accuracy of our numerical approach.

Next, we considered the effects of a single pin and a pair of pins on the liquid-state properties. The single-pin calculation was done to fix the value of the parameter $\alpha$ of Eq. (3). Using $r_{0}=0.1 a_{0}(\simeq 60 \AA$ for $B=2 \mathrm{kG}$ ), we find that in the temperature range of interest $(T \approx 15-25 \mathrm{~K})$, the integrated density inside the range of the pinning potential is close to one if $\alpha \approx 0.05-0.06$ $\left[V_{0}(r=0) \approx 7-9\right]$. The density distribution near the center of the defect is Gaussian, $\rho(r) \propto \exp \left(-\alpha \Gamma r^{2} / r_{0}^{2}\right)$, expected from Eq. (3). Since pinning of multiple vortices at radiation-induced defects is not found in experiments, higher values of $\alpha$ were not considered. Our two-pin calculations show [18] the expected oscillatory behavior [5] of the free energy as a function of the distance between the pins.

To study melting in the presence of a commensurate, periodic array of pins, we considered a triangular lattice of pins with spacing equal to $l a_{m}$, where $l$ is an integer. Thus the pin concentration is $c \equiv 1 / l^{2}$. The computational cell 


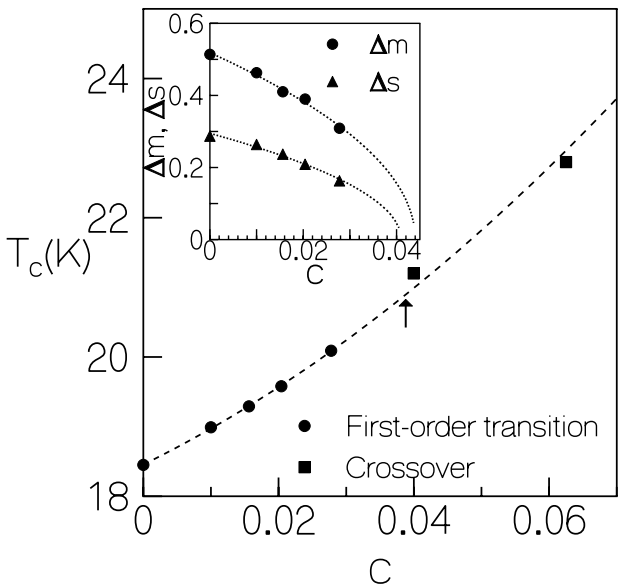

FIG. 1. The phase diagram ( $T_{c}$ vs pin concentration $\left.c\right)$ for $B=2 \mathrm{kG}$. The dashed line is a polynomial fit. Inset: Entropy change $\Delta s$ (in units of $k_{B}$ ) and order parameter jump $\Delta m$ vs $c$. Dotted lines are fits to the form const $\times\left(c_{c}-c\right)^{1 / 2}$. The approximate position of the critical point is indicated by the arrow.

used was one unit cell of the pin lattice (which contains $l^{2}$ unit cells of the vortex lattice) with one pin located at the center of the first unit cell of the vortex lattice. The value of $h$ was fixed at $a_{m} / 64$. The crystalline (or liquid) minimum of the free energy was located by starting the minimization from a crystalline (or liquidlike) initial state, usually that obtained at a nearby $T$. For both values of $B$ and smaller $c(l=10,8,7$, and 6$)$, we found a first-order transition (two distinct local minima whose free energies cross at the transition temperature). Results for $B=2 \mathrm{kG}$ are shown in Fig. 1. As expected, the presence of columnar pins increases $T_{c}$. The discontinuities in $s$ and $m$ decrease as $c$ increases (inset of Fig. 1) because pinning-induced order in the liquid increases with $c$. The results for $B=3 \mathrm{kG}$ are very similar, with $T_{c}$ reduced by approximately $3.4 \mathrm{~K}$ for all these values of $c$.

In Fig. 2, we have shown the variation of the local density $\rho$ along a line joining two neighboring pinning centers for the coexisting crystalline and liquid minima at the transition temperature for $c=1 / 64$ and $B=2 \mathrm{kG}$. This plot illustrates the ability of our numerical method to provide detailed information about the density distribution in inhomogeneous states. In the liquid state, we find [18] the expected sixfold angular modulation [5] of the density.

At larger values of $c(l<6)$, the behavior found (for both values of $B$ ) is significantly different. Here, the apparent minima obtained in "heating" runs (increasing $T$ in steps from a crystalline state at low $T$ and using the minimum obtained for the last $T$ as the input for the current minimization) and in "cooling" runs (decreasing $T$ in steps from a liquid state at high $T$ ) have almost the same free energy, but significantly different density distributions [18] and values of $m$. At $c=1 / 25(l=5)$, the difference in the values of $m$ peaks at $T=T_{x} \simeq 21.2 \mathrm{~K}$ for $B=2 \mathrm{kG}$, as shown in Fig. 3. These results suggest that

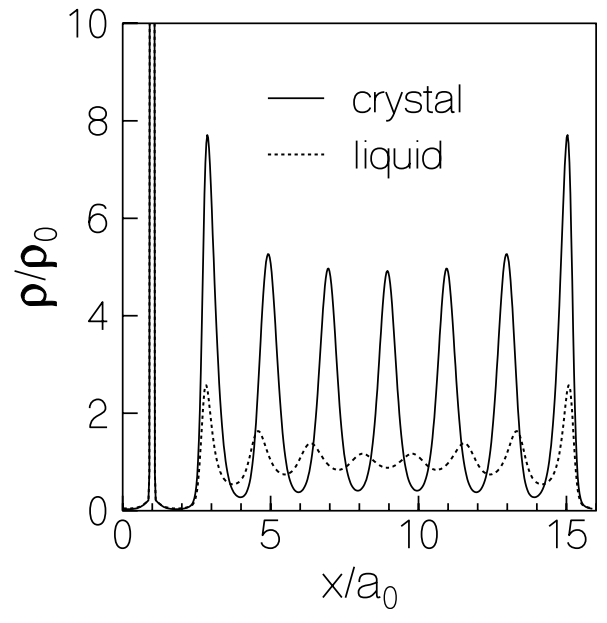

FIG. 2. The normalized local density $\rho / \rho_{0}$ along a line joining two adjacent pinning centers. Data for the coexisting crystal (solid line) and liquid (dotted line) for $c=1 / 64, B=2 \mathrm{kG}$ are shown. The peak near $x / a_{0}=1$ is at the location of a pinning center.

$F$ has only one minimum that becomes very "flat" near $T=T_{x}$ : our minimization routine, which assumes that a minimum has been reached when the gradient of the free energy becomes smaller than a small convergence parameter, stops at slightly different points when approaching a very flat minimum from different directions. To check this, we have calculated the free energy of configurations $\left\{\rho_{i}(x)\right\}$ defined by $\rho_{i}(x)=x \rho_{i}^{(1)}+(1-x) \rho_{i}^{(2)}$, where $\left\{\rho_{i}^{(1)}\right\}$ and $\left\{\rho_{i}^{(2)}\right\}$ are the configurations obtained in heating (crystal) and cooling (liquid) runs, respectively, and $0 \leq x \leq 1$ is a mixing parameter. A plot of the free energy as a function of $x$ [or of $m(x)=x m^{(1)}+(1-x) m^{(2)}$, where $m^{(1)}$ and $m^{(2)}$ are the order parameters in the two configurations] exhibits a minimum at $x=x_{0} \sim 0.5$ at all temperatures. A typical plot, for $B=2 \mathrm{kG}, T=21.2 \mathrm{~K}$,

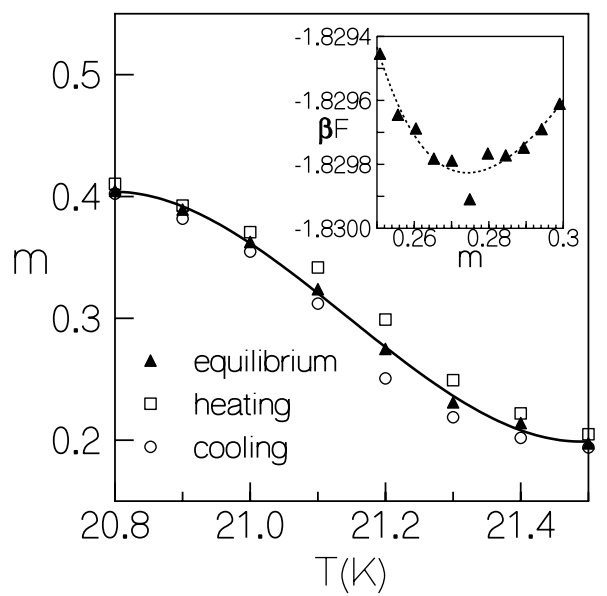

FIG. 3. The "heating," "cooling," and "equilibrium" values of the order parameter $m$ (see text) as functions of $T$ for $c=1 / 25$, $B=2 \mathrm{kG}$. The solid line is a polynomial fit to the equilibrium data. Inset: Plot of the free energy vs $m(x)$ at $T=21.2 \mathrm{~K}$. The dotted line is the best fit to Eq. (4). 
is shown in the inset of Fig. 3. In contrast, similar plots for smaller values of $c$ exhibit a clear maximum at an intermediate value of $x$. These results confirm that, for $c=1 / 25$ or more, the free energy has a unique minimum that lies between the configurations obtained in heating and cooling runs. Thus, no first-order transition occurs at $c=1 / 25$, and the line of first-order transitions found for smaller values of $c$ must end at a critical point lying between $c=1 / 36$ and $c=1 / 25$. At $c=1 / 25$, the change from liquidlike to solidlike behavior occurs as a sharp crossover. We identify the temperature $T_{x}$, which coincides with the temperature at which the temperature derivative of the "equilibrium" value, $m\left(x_{0}\right)$, of the order parameter peaks, as the crossover temperature. The sharpness of the observed crossover (see Fig. 3) suggests that $c=1 / 25, T=T_{x} \simeq 21.2 \mathrm{~K}$ is close to the critical point for $B=2 \mathrm{kG}$, as shown in Fig. 1. For $c=1 / 16$ the crossover is smoother. Our results for $B=3 \mathrm{kG}$ are very similar, with $T_{x} \simeq 17.7 \mathrm{~K}$ for $c=1 / 25$. The critical point should be experimentally accessible: The pin lattice spacing for $B=2 \mathrm{kG}, c=1 / 25$ should be $\sim 55 \mu \mathrm{m}$, close to the spacing of the radiation-induced pin array of Ref. [6]. The same group recently showed [19] that columnar pins can be created in BSCCO in a highly controlled manner.

We can describe the basic physics underlying this phase diagram as follows. In the presence of commensurate periodic pinning the liquid and the crystal have the same symmetry. Since the degree of order in the liquid increases with $c$, the liquid and the crystal become indistinguishable beyond a critical value of $c$. Thus, it is possible to go from one phase to the other without crossing a phase boundary. A simple Landau theory illustrates this. Symmetry considerations [20] suggest the following Landau expansion for F:

$$
\beta F=\frac{1}{2} a_{2} m^{2}-\frac{1}{3} a_{3} m^{3}+\frac{1}{4} a_{4} m^{4}-h m,
$$

where $a_{3}$ and $a_{4}$ are positive constants, $a_{2}$ is a decreasing function of temperature, and the "ordering field" $h$ is proportional to the pin concentration $c$. This free energy leads to a first-order transition for $h<h_{c}=a_{3}^{3} / 27 a_{4}^{2}$ and a critical point at $h=h_{c}, a_{2}=a_{2 c}=a_{3}^{2} / 3 a_{4}$. The transition temperature increases with $h$, and the latent heat and $\Delta m$ vanish as $\left(h_{c}-h\right)^{1 / 2}$ as $h$ approaches $h_{c}$ from below. As shown in Fig. 1 (inset), our data for $\Delta s$ and $\Delta m$ are well described by the form $\propto\left(c_{c}-c\right)^{1 / 2}$ with $c_{c}$ close to $1 / 25$. For a more quantitative comparison, we have fitted the $\beta F$ vs $m$ data for $B=2 \mathrm{kG}, T=21.2 \mathrm{~K}$ to the form of Eq. (4) (inset of Fig. 3). The values of $a_{2 c}$ and $h_{c}$, calculated from the best-fit values of $a_{3}$ and $a_{4}$, are [18] less than $1.5 \%$ lower than the best-fit values of $a_{2}$ and $h$, indi- cating that the critical point for $B=2 \mathrm{kG}$ is very close to $c=1 / 25, T=21.2 \mathrm{~K}$. This explains the sharpness of the crossover at $c=1 / 25$. Our results for $B=3 \mathrm{kG}$ are very similar. The simple Landau theory, thus, provides a semiquantitative account of our density functional results and confirms that a critical point lies very close to $c=1 / 25$ for both values of $B$. This critical point is analogous to the liquid-gas critical point in mean-field theory. Fluctuations are expected to change this correspondence because the symmetry of our order parameter is different from that for the liquid-gas transition. However, the first-order melting line and the existence and experimental accessibility of the critical point should be quantitatively described by our work.

Thus, we predict here that vortex lattice melting in BSCCO with commensurate periodic pinning will exhibit an accessible critical point. Experiments to verify this prediction would be most welcome. Our results should apply with little change to thin-film samples, where $\tilde{C}$ is very similar [5]. Applications to other systems [14,15] would also be of interest.

*Also at CMTU, JNCASR, Bangalore 560064, India. ${ }^{\dagger}$ Electronic address: otvalls@tc.umn.edu

[1] L. Civale et al., Phys. Rev. Lett. 67, 648 (1991).

[2] R. C. Budhani, M. Suenaga, and S.H. Liou, Phys. Rev. Lett. 69, 3816 (1992).

[3] D. R. Nelson and V. M. Vinokur, Phys. Rev. B 48, 13060 (1993).

[4] L. Radzihovsky, Phys. Rev. Lett. 74, 4923 (1995).

[5] C. Dasgupta and D. Feinberg, Phys. Rev. B 57, 11730 (1998).

[6] K. Harada et al., Science 274, 1167 (1996).

[7] M. Baert et al., Phys. Rev. Lett. 74, 3269 (1995).

[8] G. Blatter et al., Rev. Mod. Phys. 66, 1125 (1994).

[9] Y. Y. Goldschmidt, Phys. Rev. B 56, 2800 (1997).

[10] T. V. Ramakrishnan and M. Yussouff, Phys. Rev. B 19, 2775 (1979).

[11] S. Sengupta et al., Phys. Rev. Lett. 67, 3444 (1991); G. I. Menon et al., Phys. Rev. B 54, 16192 (1996).

[12] C. Dasgupta and O. T. Valls, Phys. Rev. E 62, 3648 (2000).

[13] D. R. Nelson and B. I. Halperin, Phys. Rev. B 19, 2457 (1979).

[14] Q.-H. Wei et al., Phys. Rev. Lett. 81, 2606 (1998).

[15] E. R. Dufresne et al., cond-mat/0008414.

[16] J. P. Hansen and I. R. McDonald, Theory of Simple Liquids (Academic, London, 1986).

[17] F. Pinski (private communication).

[18] C. Dasgupta and O. T. Valls (to be published).

[19] A. Tonomura et al., Nature (London) 412, 620 (2001).

[20] S. Alexander and J. McTague, Phys. Rev. Lett. 41, 702 (1978). 